\title{
Memory effect on the multiphoton coherent destruction of tunneling in the electron transport of nanoscale systems driven by a periodic field: A generalized Floquet approach
}

\author{
Tak-San Ho, ${ }^{1, *}$ Shih-Han Hung, ${ }^{2}$ Hsing-Ta Chen, ${ }^{2}$ and Shih-I Chu ${ }^{2,3, \uparrow}$ \\ ${ }^{1}$ Department of Chemistry, Princeton University, Princeton, New Jersey 08544, USA \\ ${ }^{2}$ Department of Physics, Center for Quantum Science and Engineering, National Taiwan University, Taipei 106, Taiwan \\ ${ }^{3}$ Department of Chemistry, University of Kansas, Lawrence, Kansas 66045, USA
}

(Received 28 April 2009; revised manuscript received 2 June 2009; published 22 June 2009)

\begin{abstract}
Time-dependent electron-transport processes are often studied in the wide-band limit. In this paper, a generalized Floquet approach beyond the wide-band limit is developed for the general treatment of memory effect on the virtually unexplored multiphoton (MP) coherent destruction of tunneling (CDT) phenomenon of periodically driven electrode-wire-electrode nanoscale systems. As a case study, we apply the approach for a detailed analysis of the electron-transport dc current in the electrode-quantum double dot-electrode system, showing the significance of memory effect as well as illustrating the origin of the MP-CDT phenomenon.
\end{abstract}

DOI: $10.1103 /$ PhysRevB.79.235323

PACS number(s): 73.23.-b, 73.63.-b, 72.10.Bg

\section{INTRODUCTION}

An accurate account of electron transport of nanoscale quantum systems driven by external fields is essential to the understanding of many fundamental time-dependent processes, ${ }^{1}$ including the photon-assisted tunneling, ${ }^{2,3}$ coherent destruction of tunneling (CDT), ${ }^{4,5}$ and nonadiabatic electron pumping. ${ }^{6}$ Time-dependent electron-transport processes are often studied in the wide-band limit (WBL). ${ }^{7}$ In particular, for temporal periodic driving fields, the Floquet theory ${ }^{8,9}$ can be invoked to enable efficient computations and analysis of the transport current and the current noise. ${ }^{1,7}$ The CDT phenomenon has been studied extensively for electrontransport problems within the WBL Floquet formulation. ${ }^{10-13}$ However, the WBL approximation neglects an important fact that the electrode-wire coupling is in general energy dependent. ${ }^{14-16}$ As a result, the memory effect (the effect of energy-dependent spectral density of electrodes) on electron transport cannot be accounted for in the Floquet theorybased calculations.

In this paper, the Floquet theory is generalized beyond the WBL to include memory effect. Specifically, we make use of this generalized Floquet method to study the memory effect on the virtually unexplored multiphoton resonance CDT phenomenon, supplementing the well-studied zero-biased CDT, with the aim to facilitate the understanding and possible development of new mesoscopic optoelectronic devices. The generalized Floquet formulation is made possible by modeling the energy-dependent electrode-wire coupling (i.e., the spectral density of the electrodes) in terms of sums of Lorentzian functions. ${ }^{17,18}$ Previously, the memory effect on the CDT of zero-biased double quantum dots driven by a periodic field has been studied in the context of the same Lorentzian ansatz ${ }^{18}$ but without taking advantage of the Floquet theory. Following the seminal work of Jauho et al. on the time-dependent transport theory, ${ }^{19}$ within the framework of the nonequilibrium Green's function, ${ }^{20,21}$ and with the adoption of the Lorentzian ansatz for the electrode spectral density, we show that the underlying time-dependent singleparticle propagator for the electrode-wire-electrode system can be efficiently solved within the general framework of the
Floquet theory without resorting to the WBL. Moreover, we find that the generalized Floquet method provides a comprehensive physical picture of the multiphoton CDT phenomenon.

The paper is organized as follows. In Sec. II we describe the general formulation of memory effect for an electrodewire-electrode quantum system (using a spinless tightbinding model) in the presence of a time-dependent external field based on the Lorentzian model for the electrode spectral density. To this end, a set of coupled ordinary differential equations for the underlying single-particle propagator is derived. Section III presents the general Floquet approach beyond the wide-band limit for the electrode-double quantumdot (DQD)-electrode system driven by a periodic external field. Section IV analyzes the memory effect on the multiphoton coherent destruction of tunneling phenomena for the electron transport involving double quantum dots in the high-frequency limit. Finally, a brief summary is given in Sec. V.

\section{GENERAL FORMULATION OF MEMORY EFFECT}

Consider a nanoscale electrode-wire-electrode quantum system driven by a time-dependent external field. The corresponding single-particle Hamiltonian $H(t)$ can be written as ${ }^{11,12}$

$$
H(t)=H_{C}(t)+H_{L}+H_{R}+H^{\prime},
$$

where $H_{C}(t)$ is the externally driven central wire Hamiltonian, $H_{L}$ and $H_{R}$ are, respectively, the left and right electrode Hamiltonians, and $H^{\prime}$ is the interaction between the wire and the electrodes. In the spinless tight-binding approximation $^{12}$ with $N$ orbitals (Fig. 1) driven by a timedependent external field, the central quantum wire Hamiltonian $H_{C}(t)$ may be expressed in terms of single-electron states $|\alpha\rangle$ 's,

$$
H_{C}(t)=\sum_{\alpha=1}^{N} \sum_{\beta=1}^{N} H_{\alpha \beta}(t)|\alpha\rangle\langle\beta|,
$$

where the wire contains $N$ sites (orbitals) designated as $1,2, \ldots, N-1, N$; the site 1 is in contact with the electrode $L$ 


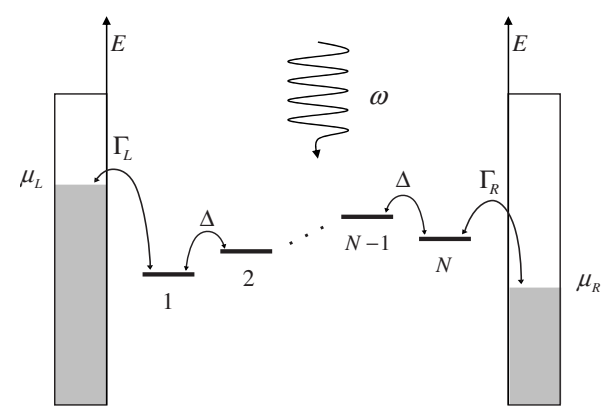

FIG. 1. Schematic diagram of an electrode- $N$-site quantum wireelectrode nanoscale system driven by a time-dependent external field of frequency $\omega$. The electrode-wire couplings are denoted by $\Gamma_{L}$ and $\Gamma_{R}$ while the site-to-site tunneling is denoted by $\Delta$. The electrochemical potentials of the left and right electrodes are denoted as $\mu_{L}$ and $\mu_{R}$, respectively.

on the left-hand side and the site $N$ is with the electrode $R$ on the right-hand side. The left and right electrode Hamiltonians $H_{L}$ and $H_{R}$ can be written as

$$
H_{L}=\sum_{q} \epsilon_{q}|L q\rangle\langle L q|
$$

and

$$
H_{R}=\sum_{q} \epsilon_{q}|R q\rangle\langle R q|
$$

respectively, in terms of the electron states $|L q\rangle$ 's and $|R q\rangle$ 's. Finally, the spectral densities of the left and right electrodes (taking into account the two spin states of electrons) can be written as

$$
\bar{\Gamma}_{L / R}(\epsilon)=4 \pi \sum_{q}\left|V_{L / R q}\right|^{2} \delta\left(\epsilon-\epsilon_{q}\right)
$$

which in turn lead to the electrode-wire coupling Hamiltonian

$$
H^{\prime}=\sum_{q}\left(V_{L q}|L q\rangle\left\langle 1\left|+V_{R q}\right| R q\right\rangle\langle N|\right)+\text { H.c. }
$$

with $V_{L q}$ and $V_{R q}$ being, respectively, coupling amplitudes between the sites $|1\rangle,|N\rangle$, and their contacting electrodes.

The underlying time-dependent equation for the singleparticle propagator $U\left(t, t_{0}\right)$ of the $N$-site quantum wire can be written in matrix form, ${ }^{12}$

$$
\imath \hbar \frac{\partial}{\partial t} U\left(t, t_{0}\right)=H_{C}(t) U\left(t, t_{0}\right)-\frac{\iota}{2} \int_{t_{0}}^{t} \bar{\Gamma}\left(t-t^{\prime}\right) U\left(t^{\prime}, t_{0}\right) d t^{\prime},
$$

where $U\left(t, t_{0}\right)$ is an $N \times N$ matrix function and $\bar{\Gamma}\left(t, t^{\prime}\right)$ is the corresponding response function (the memory kernel),

$$
\bar{\Gamma}\left(t-t^{\prime}\right)=\bar{\Gamma}_{L}\left(t-t^{\prime}\right)|1\rangle\left\langle 1\left|+\bar{\Gamma}_{R}\left(t-t^{\prime}\right)\right| N\right\rangle\langle N|,
$$

in which $\bar{\Gamma}_{L / R}\left(t-t^{\prime}\right)$ are the Fourier transforms of the spectral densities $\bar{\Gamma}_{L / R}(\epsilon)$ via the relation

$$
\bar{\Gamma}_{L / R}\left(t-t^{\prime}\right)=\frac{1}{2 \pi \hbar} \int \bar{\Gamma}_{L / R}(\epsilon) e^{-l \epsilon\left(t-t^{\prime}\right) / \hbar} d \epsilon
$$

In the WBL, ${ }^{11,12} \bar{\Gamma}_{L / R}(\epsilon)$ becomes independent of the energy $\epsilon$, i.e., $\bar{\Gamma}_{L / R}(\epsilon) \rightarrow \Gamma_{L / R}$, and as a result the response function $\bar{\Gamma}\left(t-t^{\prime}\right)$ can be reduced to the simple form,

$$
\bar{\Gamma}\left(t-t^{\prime}\right)=\left(\Gamma_{L}|1\rangle\left\langle 1\left|+\Gamma_{R}\right| N\right\rangle\langle N|\right) \delta\left(t-t^{\prime}\right) .
$$

The memory effect on the electron transport can be expediently studied by modeling the electrode spectral densities $\bar{\Gamma}_{L / R}(\epsilon)$ as sums of $M$ Lorentzian functions ${ }^{18}$

$$
\bar{\Gamma}_{L / R}(\epsilon)=\sum_{k=1}^{M} \frac{q_{k}^{L / R} b_{k}^{L / R}}{\left(\epsilon-\sigma_{k}^{L / R}\right)^{2}+\left(b_{k}^{L / R}\right)^{2}}<\infty,
$$

where $q_{k}^{L / R}, \sigma_{k}^{L / R}$, and $b_{k}^{L / R}$ are real positive fitting parameters chosen to mimic the smooth property of the spectral density. In the WBL (i.e., in the limit of the spectral widths $b_{k}^{L / R}$ $\rightarrow \infty)$, the spectral densities become $\epsilon$ independent,

$$
\lim _{b_{k}^{L / R} \rightarrow \infty} \bar{\Gamma}_{L / R}(\epsilon) \rightarrow \Gamma_{L / R}=\sum_{k=1}^{M} \frac{q_{k}^{L / R}}{b_{k}^{L / R}}<\infty .
$$

Here we assume that the ratios $q_{k}^{L / R} / b_{k}^{l / R}$ are of finite magnitude in the Lorentzian ansatz, cf. Eqs. (11) and (12), so that the values of the electrode spectral densities $\bar{\Gamma}_{L / R}(\epsilon)$ also maintain finite in the calculations.

Using the ansatz of $M$ Lorentzian spectral-density functions, cf. Eq. (11), and carrying out the integral in Eq. (9), we immediately obtain the following closed form for the response functions associated with the left and right electrodes,

$$
\bar{\Gamma}_{L / R}\left(t-t^{\prime}\right)=\frac{1}{2 \hbar} \sum_{k=1}^{M} q_{k}^{L / R} e^{-l\left(\sigma_{k}^{L / R}-l b_{k}^{L / R}\right)\left(t-t^{\prime}\right) / \hbar} .
$$

Furthermore, by making use of Eq. (13), we can recast Eq. (7) into a set of $N+2 M$ coupled ordinary differential equations

$$
\begin{gathered}
\frac{\partial U\left(t, t_{0}\right)}{\partial t}=\frac{1}{\imath \hbar}\left[H_{C}(t) U\left(t, t_{0}\right)+\sum_{k=1}^{M} \frac{l}{2} \sqrt{Q_{k}} Y_{k}\left(t, t_{0}\right)\right], \\
\frac{\partial Y_{k}\left(t, t_{0}\right)}{\partial t}=\frac{1}{\imath \hbar}\left[-\frac{l}{2} \sqrt{Q_{k}^{\mathrm{T}}} U\left(t, t_{0}\right)+\left(\boldsymbol{\sigma}_{k}-\imath B_{k}\right) Y_{k}\left(t, t_{0}\right)\right],
\end{gathered}
$$

subject to the initial conditions $U\left(t_{0}, t_{0}\right)=\mathbf{1}$ (the $N \times N$ identity matrix) and $Y_{k}\left(t_{0}, t_{0}\right)=\mathbf{0}$ (the $2 \times N$ zero matrix), where $Y_{k}\left(t, t_{0}\right), k=1, \ldots, M$ are $2 \times N$ rectangular matrix functions of the form

$$
Y_{k}\left(t, t_{0}\right)=-\frac{\sqrt{Q_{k}^{\mathrm{T}}}}{2 \hbar} \int_{t_{0}}^{t} e^{-l\left(\boldsymbol{\sigma}_{k}-l B_{k}\left(t-t^{\prime}\right)\right.} U\left(t^{\prime}, t_{0}\right) d t^{\prime},
$$

with $Q_{k}, \boldsymbol{\sigma}_{k}$, and $B_{k}$ being, respectively, $N \times 2,2 \times 2$, and 2 $\times 2$ matrices, namely, 


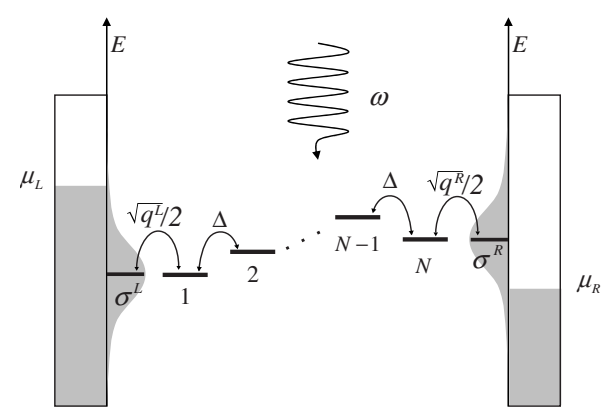

FIG. 2. Schematic diagram of an effective $(N+2)$-level electrode- $N$-site quantum wire-electrode nanoscale system driven by a time-dependent external field of frequency $\omega$ and with the electrode spectral densities $\bar{\Gamma}_{L / R}(\epsilon)$ modeled by single Lorentzian functions. The electrode-wire couplings are denoted by $\sqrt{q^{L}} / 2$ and $\sqrt{q^{R}} / 2$ while the site-to-site tunneling is denoted by $\Delta$. The electrochemical potentials of the left and right electrodes are denoted as $\mu_{L}$ and $\mu_{R}$, respectively.

$$
Q_{k}=\left(\begin{array}{cc}
q_{k}^{L} & 0 \\
0 & 0 \\
\vdots & \vdots \\
0 & 0 \\
0 & q_{k}^{R}
\end{array}\right), \quad \sigma_{k}=\left(\begin{array}{cc}
\sigma_{k}^{L} & 0 \\
0 & \sigma_{k}^{R}
\end{array}\right), \quad B_{k}=\left(\begin{array}{cc}
b_{k}^{L} & 0 \\
0 & b_{k}^{R}
\end{array}\right) \text {, }
$$

and $Q_{k}^{\mathrm{T}}$ being the matrix transpose of $Q_{k}$. Coupled equations (14) and (15) may be effectively seen as an $(N+2 M)$-level quantum system composed of an externally driven $\mathrm{N}$-site quantum wire simultaneously coupled to $M$ noninteracting two levels, as depicted in Fig. 2 for the $M=1$ case. Each two level is composed of a left $(L)$ level and a right $(R)$ level, respectively, endowed with the energies $\sigma_{k}^{L / R}$ and widths $b_{k}^{L / R}$ with $k=1, \ldots, M$. The coupling between the quantum wire and the $k$ th two levels is proportional to the square root of the fitting parameters $q_{k}^{L}$ and $q_{k}^{R}$, which scale linearly in terms of the spectral widths $b_{k}^{L}$ and $b_{k}^{R}$, respectively (by keeping the ratios $q_{k}^{L / R} / b_{k}^{l / R}$ finite). It should be pointed out that Eqs. (14) and (15) not only address the memory effect but also are applicable to both periodically and nonperiodically driven electron-transport problems. The physical quantities of interest in the electron-transport calculations can be derived in terms of the single-particle propagator $U\left(t, t_{0}\right)$ in Eqs. (14) and (15). Specifically, the single-electron retarded Green's function $G\left(t, t_{0}\right)$ can be computed from the relation ${ }^{11,12,19}$

$$
G(t, \epsilon)=\int_{0}^{\infty} e^{\imath \epsilon \tau / \hbar} U(t, t-\tau) d \tau,
$$

and for periodically driven quantum wires, $H_{C}(t+T)=H_{C}(t)$, the Fourier components

$$
G^{(k)}(\epsilon)=\frac{1}{T} \int_{0}^{T} G(t, \epsilon) e^{\imath k \omega t} d t
$$

of the periodical function $G(t, \epsilon)=G(t+T, \epsilon)$. Accordingly, the time-ensemble averaged dc electron-transport current,

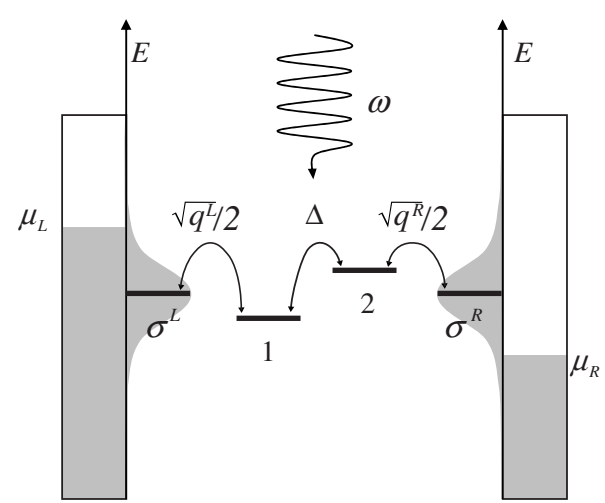

FIG. 3. Schematic diagram of an effective four-level electrodeDQD-electrode nanoscale system driven by a time-dependent external field of frequency $\omega$ and with the electrode spectral densities $\bar{\Gamma}_{L / R}(\epsilon)$ modeled by single Lorentzian functions. The electrode-wire couplings are denoted by $\sqrt{q^{L}} / 2$ and $\sqrt{q^{R}} / 2$ while the site-to-site tunneling is denoted by $\Delta$. The electrochemical potentials of the left and right electrodes are denoted as $\mu_{L}$ and $\mu_{R}$, respectively.

$$
\bar{I} \equiv \frac{1}{T} \int_{0}^{T}\left\langle I_{L}(t)\right\rangle d t
$$

can in turn be evaluated via the equation, ${ }^{12}$

$$
\bar{I}=\frac{e}{2 \pi \hbar} \sum_{k=-\infty}^{+\infty} \int d \epsilon\left\{T_{L R}^{(k)}(\epsilon) f_{R}(\epsilon)-T_{R L}^{(k)}(\epsilon) f_{L}(\epsilon)\right\},
$$

where $T_{L R}^{(k)}(\epsilon)=(1 / 4) \bar{\Gamma}_{L}(\epsilon+k \hbar \omega) \bar{\Gamma}_{R}(\epsilon)\left|G_{1 N}^{(k)}(\epsilon)\right|^{2}$ and $T_{R L}^{(k)}(\epsilon)$ $=(1 / 4) \bar{\Gamma}_{R}(\epsilon+k \hbar \omega) \bar{\Gamma}_{L}(\epsilon)\left|G_{N 1}^{(k)}(\epsilon)\right|^{2}$ are, respectively, the rightto-left and left-to-right transmission coefficients. The Fermienergy functions are $f_{L / R}(\epsilon)=1 /\left\{1+e^{\beta\left(\epsilon-\mu_{L / R}\right)}\right\}$ and the initial density matrix describing the state of the electrodes is $\rho_{0}$ $\sim e^{-\beta\left(H_{L L}+H_{R R^{-}} \mu_{L} N_{L^{-}} \mu_{R} N_{R}\right)}$, where $\mu_{L}$ and $\mu_{R}$ are, respectively, the electrochemical potentials of the left and right electrodes, $N_{L / R}$ are the numbers of electrons in the electrodes, and $\beta$ $=1 / k_{B} \mathcal{T}$ is the product of the Boltzmann constant $k_{B}$ and the temperature $\mathcal{T}$.

\section{GENERALIZED FLOQUET APPROACH BEYOND THE WIDE-BAND LIMIT}

For illustration, consider a periodically driven electrodeDQD-electrode quantum system (see Fig. 3), for which the electrode spectral densities $\bar{\Gamma}_{L / R}(\epsilon)$ may be effectively modeled by single Lorentzian functions, ${ }^{17,18}$

$$
\bar{\Gamma}_{L / R}(\epsilon)=q^{L / R} b^{L / R} /\left\{\left(\epsilon-\sigma^{L / R}\right)^{2}+\left(b^{L / R}\right)^{2}\right\} .
$$

The formulations in this section can be readily extended for the cases involving any finite number of Lorentzian functions. From Eqs. (14) and (15), it is seen that the current case reduces to a four-level quantum system governed by the time-dependent equation

$$
\frac{d}{d t}\left(\begin{array}{l}
U\left(t, t_{0}\right) \\
Y\left(t, t_{0}\right)
\end{array}\right)=\frac{1}{\imath \hbar} \mathcal{H}(t)\left(\begin{array}{l}
U\left(t, t_{0}\right) \\
Y\left(t, t_{0}\right)
\end{array}\right),
$$

where 


$$
Y\left(t, t_{0}\right)=-\frac{\sqrt{Q}}{2 \hbar} \int_{t_{0}}^{t} e^{-l\left(\boldsymbol{\sigma}-l B\left(t-t^{\prime}\right)\right.} U\left(t^{\prime}, t_{0}\right) d t^{\prime},
$$

and the effective $4 \times 4$ Hamiltonian of the electrode-DQDelectrode quantum system takes on the expression,

$$
\mathcal{H}(t)=\left(\begin{array}{cc}
H_{C}(t) & \imath \sqrt{Q} / 2 \\
-\imath \sqrt{Q} / 2 & \boldsymbol{\sigma}-\imath B
\end{array}\right) .
$$

Here, the Hamiltonian of the periodically driven DQD is given as

$$
H_{C}(t)=\left(\begin{array}{cc}
-\frac{E_{0}+A \cos \omega t}{2} & -\Delta \\
-\Delta & +\frac{E_{0}+A \cos \omega t}{2}
\end{array}\right),
$$

in which the energy difference $E(t)=E_{0}+A \cos \omega t$ shifts periodically with a frequency $\omega$ and amplitude $A$. Moreover, the electrode-DQD coupling can be written as

$$
\sqrt{Q}=\sqrt{q^{L}}|1\rangle\left\langle 1\left|+\sqrt{q^{R}}\right| 2\right\rangle\langle 2|,
$$

while the positions and widths of the left and right Lorentzian spectral-density functions are, respectively, given as

$$
\boldsymbol{\sigma}=\sigma^{L}|1\rangle\left\langle 1\left|+\sigma^{R}\right| 2\right\rangle\langle 2|,
$$

and

$$
B=b^{L}|1\rangle\left\langle 1\left|+b^{R}\right| 2\right\rangle\langle 2| .
$$

By invoking the Floquet theory, ${ }^{8,9}$ a solution $\mathcal{F}(t)$ of Eq. (23) may be expressed as

$$
\mathcal{F}(t)=\Phi(t) e^{-\imath \Lambda t / \hbar},
$$

where $\Phi(t)$ is a $4 \times 4$ matrix of periodic functions of time $t$, i.e., $\Phi(t+T)=\Phi(t)$ and $\Lambda$ is a $4 \times 4$ diagonal matrix of complex numbers satisfying the quasienergy eigenvalue equation

$$
\left\{\mathcal{H}(t)-\imath \hbar \frac{d}{d t}\right\} \Phi(t)=\Phi(t) \Lambda .
$$

From Eqs. (30) and (31), it can be shown that the fundamental solution $\mathcal{U}\left(t, t_{0}\right)$ of Eq. (23) may be written as ${ }^{8,9}$

$$
\mathcal{U}\left(t, t_{0}\right)=\mathcal{F}(t) \mathcal{F}^{-1}\left(t_{0}\right)=\left(\begin{array}{ll}
\mathcal{U}_{11}\left(t, t_{0}\right) & \mathcal{U}_{12}\left(t, t_{0}\right) \\
\mathcal{U}_{21}\left(t, t_{0}\right) & \mathcal{U}_{22}\left(t, t_{0}\right)
\end{array}\right),
$$

where $\mathcal{F}^{-1}\left(t_{0}\right)$ is the inverse of $\mathcal{F}\left(t_{0}\right)$, and $\mathcal{U}_{11}, \mathcal{U}_{21}, \mathcal{U}_{12}$, and $\mathcal{U}_{22}$ are $2 \times 2$ subblocks. The single-particle propagator $U\left(t, t_{0}\right)$ can be readily identified as

$$
U\left(t, t_{0}\right)=\mathcal{U}_{11}\left(t, t_{0}\right) U\left(t_{0}, t_{0}\right)+\mathcal{U}_{12} Y\left(t_{0}, t_{0}\right)=\mathcal{U}_{11}\left(t, t_{0}\right),
$$

which is in turn used to compute the current $\bar{I}$, cf. Eqs. (18)-(21).

On the other hand, the single-particle propagator $U\left(t, t_{0}\right)$ may be expediently written as the product

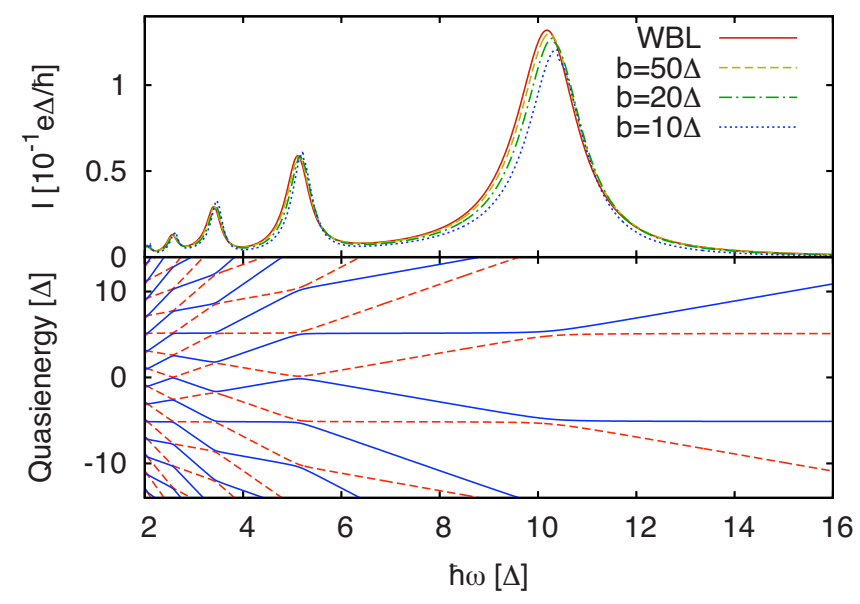

FIG. 4. (Color online) Current $\bar{I}$ and quasienergies $\lambda_{1 / 2,0}^{C}$ as functions of $\hbar \omega$ (in $\Delta$ ). $E_{0}=10 \Delta=1 \mathrm{eV}, A=6 \Delta, \sigma^{L}=\sigma^{R}=0, q^{L}=q^{R}=q$, $b^{L}=b^{R}=b$, and $\Gamma_{L}=\Gamma_{R}=q / b=0.5 \Delta$. Upper panel: the peaks of the current $\bar{I}$ from the right to left, respectively, correspond to one-, two-, three-, and four-photon resonances. Lower panel: solid (-) curves $\lambda_{1,0}^{C}$ and dashed (- - ) curves $\lambda_{2,0}^{C}$.

$$
U\left(t, t_{0}\right)=\Phi_{C}(t) X\left(t, t_{0}\right),
$$

where $\Phi_{C}(t+T)=\Phi_{C}(t)$, such that $X\left(t, t_{0}\right)$ and $Y\left(t, t_{0}\right)$ together satisfy the equation,

$$
\frac{d}{d t}\left(\begin{array}{c}
X\left(t, t_{0}\right) \\
Y\left(t, t_{0}\right)
\end{array}\right)=\frac{1}{\imath \hbar}\left(\begin{array}{cc}
\Lambda_{C} & \frac{l}{2} \Phi_{C}^{\dagger}(t) \sqrt{Q} \\
-\frac{l}{2} \sqrt{Q} \Phi_{C}(t) & \boldsymbol{\sigma}-\imath B
\end{array}\right)\left(\begin{array}{l}
X\left(t, t_{0}\right) \\
Y\left(t, t_{0}\right)
\end{array}\right),
$$

in the quasienergy (Floquet) state representation. Here the $2 \times 2$ diagonal quasienergy matrix,

$$
\Lambda_{C}=\lambda_{1,0}^{C}|1\rangle\left\langle 1\left|+\lambda_{2,0}^{C}\right| 2\right\rangle\langle 2|,
$$

contains the DQD quasienergies $\lambda_{1,0}^{C}$ and $\lambda_{2,0}^{C}$ belonging to the quasienergy states $\left|\lambda_{1,0}^{C}\right\rangle$ and $\left|\lambda_{2,0}^{C}\right\rangle$, respectively. The quasienergy state function $\Phi_{C}(t)$ associated with $\Lambda_{C}$ satisfies the quasienergy eigenvalue equation

$$
\left\{H_{C}(t)-\imath \hbar \frac{d}{d t}\right\} \Phi_{C}(t)=\Phi_{C}(t) \Lambda_{C},
$$

governing the time evolution of the DQD driven by the periodic field. The DQD quasienergy state function $\Phi_{C}(t)$ contains the elements

$$
\left(\Phi_{C}\right)_{\alpha^{\prime} \alpha}(t)=\sum_{k=-\infty}^{\infty}\left\langle\alpha^{\prime} k \mid \lambda_{\alpha, 0}^{C}\right\rangle e^{+i k \omega t},
$$

where $\alpha, \alpha^{\prime}=1,2$ and the summation is performed over the Fourier components of the field-dressed Floquet states $\left|\lambda_{1,0}^{C}\right\rangle$ and $\left|\lambda_{2,0}^{C}\right\rangle$. Figure 4 shows that the peaks of the averaged current $\bar{I}$ are closely correlated with the underlying quasienergy avoided crossing patterns at the multiphoton resonance conditions $E_{0}=n \hbar \omega, n=1,2,3,4$. Here the parameters $E_{0}$ $=10 \Delta=1 \mathrm{eV}, A=6 \Delta, \sigma^{L}=\sigma^{R}=0, q^{L}=q^{R}=q, b^{L}=b^{R}=b$, and 
$\Gamma_{L}=\Gamma_{R}=q / b=0.5 \Delta$ have been used in the calculations. Moreover, it is found that the dc current resonance peaks are blueshifted and their magnitudes slightly reduced at the decreasing spectral width $b$ of the electrodes, in accordance to the fact that a weaker electrode-wire coupling corresponds to a smaller $b(\sim q)$ value.

\section{MEMORY EFFECT ON MULTIPHOTON CDT FOR ELECTRODE-DQD-ELECTRODE SYSTEM}

To understand the memory effect on the MP-CDT phenomena, we consider the cases in the high-frequency limit and at the $n$-photon resonance condition $E_{0}=|n| \hbar \omega$. Under these situations, the corresponding generalized rotating wave approximation (GRWA) solution can be derived with the help of the transformation ${ }^{22}$

$$
\mathcal{R}(t)=e^{+l\left[E_{0} t / 2 \hbar+\phi(t)\right]}|1\rangle\left\langle 1\left|+e^{-l\left[E_{0} / 2 \hbar+\phi(t)\right]}\right| 2\right\rangle\langle 2|,
$$

where $\phi(t)=(A / 2 \hbar \omega) \sin \omega t$. In the rotated frame, the corresponding Hamiltonian of the central DQD,

$$
H_{C}^{\prime}(t)=\mathcal{R}^{\dagger}(t) H_{C}(t) \mathcal{R}(t)-\imath \hbar \mathcal{R}^{\dagger}(t) d \mathcal{R}(t) / d t,
$$

can be explicitly written as

$$
H_{C}^{\prime}(t)=-\Delta\left\{e^{-l\left[E_{0} t / \hbar+\phi(t)\right]}|1\rangle\left\langle 2\left|+e^{+l\left[E_{0} t / \hbar+\phi(t)\right]}\right| 2\right\rangle\langle 1|\right\},
$$

which in turn results in the $n$-photon GRWA Floquet Hamiltonian

$$
H_{C}^{[n]}=-\Delta \times\left|J_{n}(A / \hbar \omega)\right|(|1\rangle\langle 2|+| 2\rangle\langle 1|),
$$

where $J_{n}(x)$ is the $n$th order Bessel function of the first kind. The $n$-photon GRWA quasienergy state function

$$
\Phi_{C}^{[n]}(t)=\frac{1}{\sqrt{2}} \mathcal{R}(t)\left(\begin{array}{cc}
1 & 1 \\
-1 & 1
\end{array}\right)
$$

associated with the $n$-photon quasienergy matrix $\Lambda_{C}^{[n]}$ can be further expressed as

$$
\frac{1}{\sqrt{2}}\left(\begin{array}{cc}
\sum_{k=-\infty}^{\infty} J_{k}\left(\frac{A}{2 \hbar \omega}\right) e^{\imath k \omega t} & \sum_{k=-\infty}^{\infty} J_{k}\left(\frac{A}{2 \hbar \omega}\right) e^{\imath k \omega t} \\
-\sum_{k=-\infty}^{\infty} J_{k+n}\left(\frac{A}{2 \hbar \omega}\right) e^{-l k \omega t} & \sum_{k=-\infty}^{\infty} J_{k+n}\left(\frac{A}{2 \hbar \omega}\right) e^{-l k \omega t}
\end{array}\right),
$$

which has been derived using the well-known expansion ${ }^{22}$

$$
e^{l x \sin \omega t}=\sum_{k=-\infty}^{\infty} J_{k}(x) e^{\imath k \omega t} .
$$

It can be readily shown from Eq. (42) that the $n$-photon GRWA quasienergies are

$$
\lambda_{1,0}^{C}=-E_{0} / 2+\Delta\left|J_{n}(A / \hbar \omega)\right|,
$$

and

$$
\lambda_{2,0}^{C}=-E_{0} / 2-\Delta\left|J_{n}(A / \hbar \omega)\right|,
$$

which are separated by the Rabi oscillation frequency,

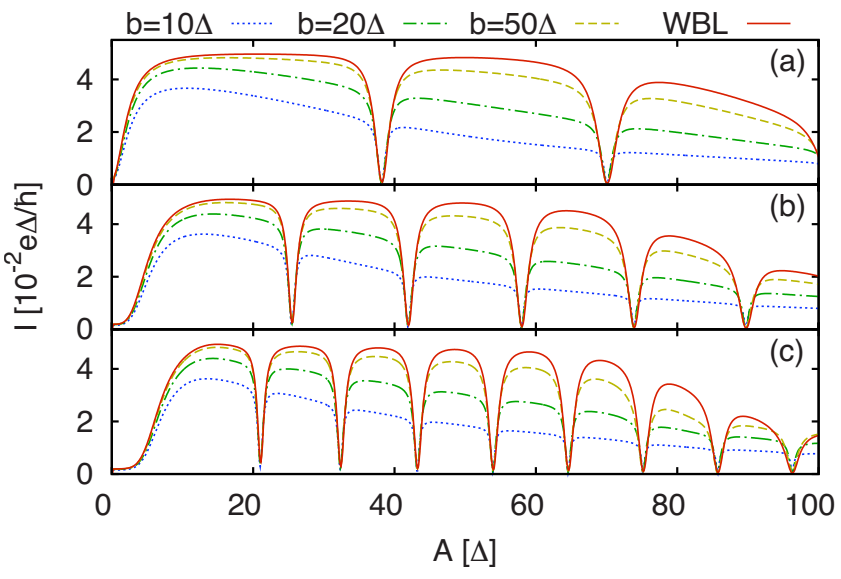

FIG. 5. (Color online) The MP-CDT at the (a) one-, (b) two-, and (c) three-photon resonances at different spectral widths $b$ and at the WBL. The abscissa is the field amplitude $A$ (in $\Delta$ ). The minima correspond to the roots of $J_{1}(A / \hbar \omega)=0$ (for one photon), $J_{2}(A / \hbar \omega)=0$ (for two photon), and $J_{3}(A / \hbar \omega)=0$ (for three photon), coinciding with the respective degenerate quasienergies $\lambda_{1,0}^{C}=\lambda_{2,0}^{C}$ $= \pm E_{0} / 2, E_{0}=10 \Delta=1 \mathrm{eV}$, where $\hbar \omega=E_{0}, E_{0} / 2, E_{0} / 3$, respectively, for one-, two-, and three-photon CDTs. See Fig. 4 for all other parameters.

$$
\Omega_{n}=2 \Delta\left|J_{n}(A / \hbar \omega)\right|,
$$

of the $n$-photon driven resonant DQD. ${ }^{22}$ Numerical results in Fig. 5, based on Eq. (23), reveals that the averaged current $\bar{I}$ is suppressed at the roots of $J_{n}(A / \hbar \omega)=0, n \geq 1$ (due to the vanishing $\Omega_{n}$ ), a clear manifestation of the MP-CDT similar to the well-known zero-biased CDT at the roots of $J_{0}(A / \hbar \omega)=0 .{ }^{10-13,18,22}$ It is found that the averaged current $\bar{I}$ in general decreases as the field amplitude increases. The memory effect (due to a decreasing spectral-density width $b$, therefore, corresponding to a deceasing electrode-wire coupling $q$ value) is to reduce the average current $\bar{I}$ but without qualitatively altering the feature of the MP-CDT. Calculations of the average current $\bar{I}$ (not shown) based on Eqs. (35) and (44) showed good agreements with the numerically exact results (Fig. 5), albeit of smaller $\bar{I}$ values (especially at the large $A$ limit). In the following, we provide a qualitative picture of the MP-CDT phenomena.

At the MP-CDT condition $\Omega_{n}=0$, cf. Eq. (48), the DQD ceases to oscillate and the corresponding quasienergy state function $\Phi_{C}^{[n]}(t)$, Eq. (44), may be further approximated by its time-averaged counterpart

$$
\bar{\Phi}_{C}^{[n]}=\frac{1}{T} \int_{0}^{T} \Phi_{C}^{[n]}(t) d t=\frac{1}{\sqrt{2}}\left(\begin{array}{cc}
J_{0}\left(\frac{A}{2 \hbar \omega}\right) & J_{0}\left(\frac{A}{2 \hbar \omega}\right) \\
-J_{n}\left(\frac{A}{2 \hbar \omega}\right) & J_{n}\left(\frac{A}{2 \hbar \omega}\right)
\end{array}\right) .
$$

It is then seen that at the weak-field amplitude, $J_{0}(A / 2 \hbar \omega)$ $\approx 1$ and $J_{n}(A / 2 \hbar \omega) \approx 0, n \geq 1$ since $J_{n}(x) \sim x^{n} / 2^{n} n$ ! for $x$ $\ll 1$, where $x=A / 2 \hbar \omega$. Therefore, the off-diagonal electrodeDQD coupling in Eq. (35) can be approximated as 


$$
\sqrt{Q} \times \bar{\Phi}_{C}^{[n]} \approx \frac{1}{\sqrt{2}}\left(\begin{array}{cc}
\sqrt{q^{L}} & \sqrt{q^{L}} \\
0 & 0
\end{array}\right),
$$

implying that the left-hand side electrode $L$ and the degenerate DQD Floquet states $\left|\lambda_{1,0}^{C}\right\rangle$ and $\left|\lambda_{2,0}^{C}\right\rangle$ form a closed current loop, which is separated from the right-hand side electrode $R$, or vice versa. In this case, corresponding to $A \ll 2 E_{0}$ $=20 \Delta$ in Fig. 5, little current is allowed to flow through the electrode-DQD-electrode quantum system, as indicated in the first minima of all panels in Fig. 5. On the other hand, at the strong-field amplitude, $\bar{\Phi}_{C}^{[n]}=0$, implying that the degenerate DQD Floquet states are decoupled from both electrodes, thus $\bar{I}=0$, as shown in the minima toward the far right end in Fig. 5 in the limit $A \gg 2 E_{0}=20 \Delta$, since $J_{n}(x)$ $\sim \sqrt{2 / \pi x} \cos [x-(2 n+1) \pi / 4]$ for $x \gg n$. However, the generally time-dependent GRWA quasienergy state matrix $\Phi_{C}^{[n]}(t)$ may not be well approximated by its time-averaged counterpart $\bar{\Phi}_{C}^{[n]}$ away from the weak-field/strong-field amplitude regimes. Consequently, the CDT phenomenon involving more than one photon (i.e., $n>1$ ) may be less pronounced at the intermediate field amplitude, as clearly shown (here $20 \Delta$ $<A<60 \Delta$ ) in the two- and three-photon CDT [the middle (b) and lower (c) panels in Fig. 5].

\section{SUMMARY}

In summary, we have presented a generalized Floquet approach beyond the wide-band limit for studying memory ef- fect on multiphoton coherent destruction of tunneling phenomena of a periodically driven electron transport of nanoscale quantum systems. The general formulation of the memory effect is equally applicable to periodically and nonperiodically driven electron-transport problems. In particular, the generalized Floquet approach can be extended to involve any number of quantum dots, including a single-quantum dot, and can be readily adopted to study a host of timedependent electron-transport processes that may be of interest in nanoscale devices. In the high-frequency limit, simple physical pictures have been given for the occurrence of the MP-CDT phenomena in the electrode-DQD-electrode quantum system. Numerical simulations at different spectral widths, as well as in the wide-band limit, showed that the memory effect reduces the electron-transport dc current without altering the feature of the MP-CDT phenomena, consistent with the Lorentzian spectral-density ansatz.

\section{ACKNOWLEDGMENTS}

This work was partially supported by the Chemical Sciences, Geosciences, and Biosciences Division of the Office of Basic Energy Sciences, Office of Sciences, Department of Energy, by the National Science Foundation. We also would like to acknowledge the partial support of the National Science Council of Taiwan (Grant No. 97-2112-M-002-003MY3) and National Taiwan University (Grant No. 97R0066).

\footnotetext{
*tsho@princeton.edu

‘sichu@ku.edu

${ }^{1}$ G. Platero and R. Aguado, Phys. Rep. 395, 1 (2004).

${ }^{2}$ C. A. Stafford and N. S. Wingreen, Phys. Rev. Lett. 76, 1916 (1996).

${ }^{3}$ C. Meyer, J. M. Elzerman, and L. P. Kouwenhoven, Nano Lett. 7, 295 (2007).

${ }^{4}$ F. Grossmann, T. Dittrich, P. Jung, and P. Hänggi, Phys. Rev. Lett. 67, 516 (1991).

${ }^{5}$ F. Großmann and P. Hänggi, Europhys. Lett. 18, 571 (1992).

${ }^{6}$ K. Saito and Y. Kayanuma, Phys. Rev. B 70, 201304(R) (2004).

${ }^{7}$ S. Kohler, J. Lehmann, and P. Haänggi, Phys. Rep. 406, 379 (2005).

${ }^{8}$ J. Shirley, Phys. Rev. 138, B979 (1965).

${ }^{9}$ S.-I. Chu and D. A. Telnov, Phys. Rep. 390, 1 (2004).

${ }^{10}$ J. Lehmann, S. Camalet, S. Kohler, and P. Hänggi, Chem. Phys. Lett. 368, 282 (2003).

${ }^{11}$ S. Camalet, J. Lehmann, S. Kohler, and P. Hänggi, Phys. Rev. Lett. 90, 210602 (2003).
}

${ }^{12}$ S. Camalet, S. Kohler, and P. Hänggi, Phys. Rev. B 70, 155326 (2004).

${ }^{13}$ K.-I. Noba, Phys. Lett. A 372, 6212 (2008).

${ }^{14}$ T. Kwapiński, R. Taranko, and E. Taranko, Phys. Rev. B 66, 035315 (2002).

${ }^{15}$ D. Hou, Y. He, X. Liu, J. Kang, J. Chen, and R. Han, Physica E (Amsterdam) 31, 191 (2006).

${ }^{16}$ P. Myöhänen, A. Stan, G. Stefanucci, and R. van Leeuwen, EPL 84, 67001 (2008).

${ }^{17}$ N. S. Wingreen and Y. Meir, Phys. Rev. B 49, 11040 (1994).

${ }^{18}$ S. Welack, M. Schreiber, and U. Kleinekathöfer, J. Chem. Phys. 124, 044712 (2006).

${ }^{19}$ A.-P. Jauho, N. S. Wingreen, and Y. Meir, Phys. Rev. B 50, 5528 (1994).

${ }^{20}$ L. P. Kadanoff and G. Baym, Quantum Statistical Mechanics (Benjamin, New York, 1962).

${ }^{21}$ L. V. Keldysh, Sov. Phys. JETP 20, 1018 (1964).

${ }^{22}$ S. Ashhab, J. R. Johansson, A. M. Zagoskin, and F. Nori, Phys. Rev. A 75, 063414 (2007). 